\title{
Neurological commentary addressing the article titled "Guidance for switching from off-label antipsychotics to pimavanserin for Parkinson's disease psychosis: an expert consensus"
}

\author{
Neal Hermanowicz (iD *
}

Department of Neurology, University of California-Irvine, Irvine, California, USA

Parkinson's disease psychosis (PDP) occurs commonly and can comprise the most troubling symptoms among the many that occur with this illness. Prior treatment options for PDP have been limited and unsatisfactory due to uneven efficacy data, burdensome monitoring, and lack of a specific FDA indication coupled with warnings of increased mortality. Pimavanserin, approved for the treatment of PDP by the FDA in 2016, overcomes some of these obstacles, with data proven efficacy and without the frequent monitoring required for clozapine. This presents an opportunity to transition patients with PDP to pimavanserin from older therapies. Black and colleagues provide their thoughtful recommendations on how to achieve this transition to pimavanserin while maintaining symptom control and minimizing disruptions that might occur with a medication change.

Received 27 June 2018; Accepted 27 June 2018

Key words: Clozapine, delusions, hallucinations, Parkinson's disease, pimavanserin, psychosis, quetiapine.

Parkinson's disease psychosis (PDP) can rapidly top the long list of symptoms that trouble people with Parkinson's disease (PD) and their spouses/caregivers. When hallucinations or delusions are frightening or threatening, they may impel the patient to call the police, protect themselves with weapons, accuse their spouses/caregivers of schemes to harm them, or even physically assault those who provide their care. When these symptoms become troubling, the care for the patient takes a turn in a direction that is decidedly more complicated and unpleasant for all involved. Some evidence indicates tha early recognition and intervention may alter the progressive natural history of PDP, and delay or prevent these symptoms from becoming so problematic. ${ }^{1}$

Reliable treatment options for PDP that do not also create secondary problems of worsening motor function or side effects have been scarce. The choices for

* Address for correspondence: Neal Hermanowicz, MD, Professor of Neurology, Director, Parkinson's Disease \& Movement Disorders Program, Department of Neurology, University of California, Irvine, 1 Medical Plaza Drive, Irvine, CA, 92697, USA.

(Email: nhermano@uci.edu) treatment increased with the arrival of pimavanserin in 2016. It was developed specifically to mimic the serotoninergic action of other antipsychotic drugs, including clozapine and quetiapine, which was believed to target PDP symptoms. Black and colleagues ${ }^{2}$ describe methods of transition to pimavanserin from another antipsychotic medication, particularly from quetiapine or clozapine, for PDP treatment, emphasizing receptor profiles, pharmacokinetics, and pharmacodynamics. The authors also draw upon prior experience with transitioning from one medication to another in other, primary psychiatric illnesses, not PDP.

Clozapine, the first atypical antipsychotic medication, has been examined in 2 clinical trials and has been found to be effective and reasonably well tolerated. The potential anticholinergic side effects mentioned by Black et al were not observed in these trials. However, despite established efficacy, clozapine has been seldom prescribed for PDP by neurologists or psychiatrists. The requirement for monitoring blood counts for the rare but possibly lethal side effect of agranulocytosis is regarded by patients, caregivers, and providers alike as excessively burdensome, particularly for people already struggling with PDP. 
Quetiapine emerged as the de facto choice for PDP. At low doses, including those generally used in treating PDP, it does not cause motor worsening, and its side effect of drowsiness is sometimes welcomed by patients and spouses suffering from the sleep disruption that is common in PD. Many clinicians, including many experienced movement disorders subspecialists (MDS), regard quetiapine as their "go to" medication to treat PDP. However, clinical trials have revealed that quetiapine is not reliable for this purpose. Black and colleagues make reference to this in their comment that 4 of 5 trials of quetiapine for PDP failed to demonstrate efficacy. Still, and somewhat puzzlingly, quetiapine appears to be the medication of choice for PDP by many MDS neurologists, who remain convinced that it works in their hands despite the absence of supporting data. This is reminiscent of the belief that anticholinergic drugs are particularly effective for the treatment of PD tremor. The troublesome side effects of anticholinergics are generally well recognized; nonetheless the tremor benefit is still advocated by some MDS experts despite a Cochrane review that failed to find evidence substantiating this notion. ${ }^{3}$ This may represent persistence of ideas emanating from the pre-levodopa era when anticholinergics were the mainstay of therapy. The use of quetiapine for PDP and anticholinergics for tremor have achieved a type of folk medicine adherence, passed down from preceding generations of neurologists. Both serve as interesting examples of persistence of older therapies when newer, more data-supported choices are available. The adoption of new therapies by physicians is itself an area of investigation. ${ }^{4}$

Pimanaserin requires special consideration due to 2 major factors. The first is its long half-life of 57 hours, compared to about 6 hours and 14 hours for quetiapine and clozapine, respectively. Serum steady state for pimavanserin may be achieved after approximately 2 weeks (pharmacokinetics). However, the full clinical benefit may not be realized for 4 weeks or more (pharmacodynamics). Consequently, if pimavanserin is to replace clozapine or quetiapine, an overlap of antipsychotic medications is recommended by Black et al for 4 weeks or more prior to withdrawing the initial drug.

The second factor is the receptor interaction profile of pimavanserin. Pimavanserin is restricted to inverse agonist activity at $5-\mathrm{HT}_{2 \mathrm{~A}}$ and to a lesser extent $5-\mathrm{HT}_{2 \mathrm{C}}$ serotonin receptors. If pimavanserin is intended to replace another antipsychotic medication with a broader receptor profile, such as quetiapine, abrupt cessation of the initial drug could result in unpleasant symptoms of withdrawal. Consequently a slow taper is recommended.

The authors emphasize that other antipsychotics with dopamine blocking activity are considered to be inappropriate choices for the treatment of PDP due to worsening of PD motor symptoms. Nonetheless, medications such as risperidone, olanzapine, aripiprazole, and ziprasidone are still sometimes prescribed for PDP by clinicians who are comfortable with these drugs but perhaps less familiar with care of patients with PD. In this scenario the motivation for a transition to pimavanserin would be greater still and conceivably involve also consideration of adjustment of PD medications when the transition is complete and the dopamine receptor antagonist has been removed.

The question of how to address the potential $\mathrm{QT}_{\mathrm{C}}$ interval prolongation with combined antipsychotic treatment is troubling and not resolved by Black and colleagues. The concern of course is $\mathrm{QT}_{\mathrm{C}}$ prolongation may lead to torsades de pointes (TdP), a ventricular arrhythmia associated with syncope and sudden death. This is complicated by several factors: the $\mathrm{QT}_{\mathrm{C}}$ interval varies with heart rate; some people have a genetic predisposition for a long $\mathrm{QT}_{\mathrm{C}}$ at baseline without medication exposure; and there is not a linear correlation of occurrence of $\mathrm{TdP}$ to the $\mathrm{QT}_{\mathrm{C}}$ interval. In addition, several common conditions have been described as risk factors for $\mathrm{QT}_{\mathrm{C}}$ prolongation, including advanced age, electrolyte abnormalities, diabetes, hypertension, and heart disease. Also, numerous other medications may cause $\mathrm{QT}_{\mathrm{C}}$ prolongation, including some that are commonly prescribed, such as citalopram, erythromycin, ciprofloxacin, and levofloxacin. Identifying people treated with an antipsychotic medication who may be at increased risk of TdP is not a simple task. The recommendation to exercise caution when overlapping antipsychotic medications is sound but vague. It is reasonable to obtain a baseline ECG in patients with known heart disease, such as myocardial infarction or prior ventricular arrhythmia, before initiating any medication that has been identified to cause $\mathrm{QT}_{\mathrm{C}}$ prolongation. Conferring with the treating cardiologist prior to initiating a medication that may lengthen the $\mathrm{QT}_{\mathrm{C}}$ interval would be wise. A $\mathrm{QT}_{\mathrm{C}}$ interval over 500 msec should give one pause about initiating a medication that may increase this.

Two central questions pertinent to switching antipsychotic medication for PDP were not addressed in the recommendations of Black and colleagues. First, what is the likelihood that switching to pimavanserin from quetiapine or clozapine will result in improved efficacy or a reduction of side effects? To date, no comparison studies between pimavanserin and quetiapine or clozapine have been published. What has been established is that pimavanserin met the US FDA requirements for approval for treatment of PDP, whereas neither quetiapine nor clozapine has such approval, and, more importantly, the efficacy studies of quetiapine for PDP have been at best inconclusive. The clinical trial results on the efficacy of quetiapine for treatment of PDP might give one pause about 
prescribing this medication that carries a boxed warning about death, as all antipsychotic medications do. Obviously a switch from clozapine to pimavanserin will eliminate the required blood tests and frequent pharmacy visits, which presumably the patient and his/her caregiver would welcome.

Second, what is the next step when PDP symptoms persist or recur and are troubling when the patient is taking $34 \mathrm{mg}$ of pimavanserin? The data from the clinical trial that the US FDA approval was based upon showed the majority of participants on active treatment with pimavanserin experienced improvement, but $26.3 \%$ did not improve or worsened. The reason for this lack of response or worsening is unclear. No difference in response to pimavanserin was observed according to participant age, gender, Mini-Mental Status Exam score (a score of 21 or higher was required for study entry), or severity of PDP upon entering the study. Although serotonin very likely plays a role in PDP, and in particular the $5-\mathrm{HT}_{2 \mathrm{~A}}$ receptor that is found with increased density in PDP patients, it is also likely that PDP is not a single neurotransmitter phenomenon. How to treat patients with persistent PDP despite maximal doses of pimavanserin or clozapine is not yet defined.

The symptoms of PDP are often disturbing, disruptive, and sometimes downright dangerous. They can add substantially to the suffering inflicted by PD, which even without PDP is an efficient tormentor of patients, literally from head to toe and day and night, which of course impacts their loved ones as well. Pimavanserin provides another opportunity to alleviate some of this suffering. Black and colleagues provide their experienced insight in transitioning to pimavanserin in a thoughtful and cautious manner.

\section{Disclosures}

Neal Hermanowicz has the following disclosure: Acadia Pharmaceuticals, speakers bureau, consultant, personal fees.

\section{REFERENCES:}

1. Goetz CG, Fan W, Leurgans S. Antipsychotic medication treatment for mild hallucinations in Parkinson's disease: positive impact on long-term worsening. Mov Disord. 2008; 23(11): 1541-1545.

2. Black KJ, Nasrallah H, Isaacson S, et al. Guidance for switching from off-label antipsychotics to pimavanserin for Parkinson's disease psychosis: an expert consensus. CNS Spectr. [under review].

3. Katzenschlager R, Sampaio C, Costa J, Lees A. Anticholinergics for symptomatic management of Parkinson's disease. Cochrane Database Syst Rev. 2003; (2): CD003735.

4. Garjón FJ, Azparren A, Vergara I, Azaola B, Loayssa JR. Adoption of new drugs by physicians: a survival analysis. BMC Health Serv Res. 2012; 12: 56 